\title{
Prevotella disiens
}

National Cancer Institute

\section{Source}

National Cancer Institute. Prevotella disiens. NCI Thesaurus. Code C86668.

A species of obligately anaerobic, Gram-negative, rod shaped bacteria assigned to the phylum Bacteroidetes. This species is nonmotile, non-spore forming, non-pigmented, indole negative, hydrolyzes starch, but not esculin, generally nonhemolytic, produces succinic and acetic acid from peptone-yeast-glucose medium, digests gelatin, milk, casein, and meat, and ferments glucose maltose, and starch, but not galactose, sucrose, lactose, or mannose. P. disiens was isolated from the oral cavity and female genital tract where it is associated with infections, but is also linked to abscesses and a variety of systemic infections. 